\title{
Evaluation Data of Dried Vegetables and Fruits
}

\author{
Necla Çağlarırmak ${ }^{1, a, *}$, Ahmet Zeki Hepçimen ${ }^{1, b}$ \\ ${ }^{1}$ Food Technology Department, Saruhanll Collage, Manisa Celal Bayar University, 45800 Saruhanll/Manisa, Turkey \\ *Corresponding author

\begin{tabular}{|c|c|}
\hline A R T I C L I N F O & B S T R A C T \\
\hline $\begin{array}{l}\text { \#This study was presented as an online } \\
\text { presentation at the } 2^{\text {nd }} \text { International } \\
\text { Journal of Agriculture - Food Science } \\
\text { and Technology (TURJAF 2021) } \\
\text { Gazimağusa/Cyprus } \\
\text { Research Article } \\
\text { Received : 01/11/2021 } \\
\text { Accepted : } 31 / 12 / 2021\end{array}$ & $\begin{array}{l}\text { Drying is the process of removing of the water that has destroying effect in food products by } \\
\text { evaporation and. Research project on the basis of direct sun drying and solar greenhouse. Basic } \\
\text { operations research in food engineering, food chemistry, food quality control and toxicology has } \\
\text { been established over such a broad spectrum. Subjects of investigation were in accordance with all } \\
\text { of the values of dry matter basis. The study of dry matter and water activity values of each product } \\
\text { (aw), direct sun drying, drying in the greenhouse. It was determined comparing nutrients of samples } \\
\text { those were applied directly to the greenhouse and drying in the sun. Sampling patterns of research } \\
\text { were explained as follow; tomatoes drying in the sun (external environment), and greenhouse, bell } \\
\text { peppers in the greenhouse and drying in the sun, soaked raisins (sultanas) and not-soaked (raisin), } \\
\text { sun-dried, sun-dried fig products directly. Nutrients of the samples such as; lycopene, thiamine } \\
\text { (B1), riboflavin (B2), retinol (A), Pyridoxine (B6), ascorbic acid (C), folic acid, magnesium (Mg), } \\
\text { potassium (K), sodium (Na), phosphorus (P), zinc (Zn), iron (Fe), copper (Cu) were quantitatively } \\
\text { determined. The red pepper products, dried figs and dried grapes mycotoxin amounts were in safe } \\
\text { levels, which had not created any hazard and risk for health. Red pepper and dried figs, total } \\
\text { aflatoxins, (B1, B2, G1, G2), ochratoxin A (OTA) levels in raisin in the European Union is set well } \\
\text { below the limits in terms of human health hazard and the risk factor has been identified. }\end{array}$ \\
\hline
\end{tabular}

\section{Introduction}

Dried or semi-dried tomatoes, dried peppers provide important inputs for the economy in Turkey's the most important exporting food products as well as in domestic market. Dried tomatoes are mostly produced in Aegean Region (Ayan and Artık, 2010; Şahin et al., 2010; Çağlarırmak and Hepçimen, 2013).

Highly hydrated agricultural products are dried for weight reduction and stabilization,

Including fruits and vegetables, meats and aromatic plants (Bonazzi and Duomolin, 2011).

The basis of food preservation and basing methods is to carry out various food processes to minimize and / or eliminate the factors that spoil the shelf life of food, to stop and slow down the reactions that spoil the food (Hui, 2006).

While performing these processes, preserving the nutritional value of food and ensuring quality, preserving and even improving its sensory properties are also considered as basic principles. Economic, environmentally friendly hybrid systems have been applied in recent years while producing dried food in high quality. (Miranda et al., 2009; Louka et al., 2004).
Mainly food preservation and preservation methods are; Pasteurization and sterilization where thermal processes are applied, freezing, drying, pickling, irradiation, high pressure applications, concentration packaging under modified atmosphere, vacuum packaging, freeze drying (Gould, 2012).

The meaning of hurdle effect is to prevent the harmful effects of microorganisms in food preservation, the main physicochemical parameters that affect the activities of microorganisms; The aim is to ensure food safety by controlling water activity aw, $\mathrm{pH}$, redox potential $\mathrm{Eh}$, high temperature $F$ value during food process and $t$ value during food storage (Singh and Shalini, 2016).

Drying is one of the oldest known methods and it is one of the food preservation methods that are easily made both in industrial and home conditions. The drying method is both economical and, in many cases, environmentally friendly, products that take up less space are obtained due to the reduction of the volume and box in the final product. Reduction in weight and volume, minimizing packaging, storage and transportation costs were obtained as a result of drying process (Okos et al., 1992). 
Little or no food additives are added during drying processes. It is simple to apply technologies and food processing that modern systems have been used in recent years (Moses, et al., 2014). Some of food protection agents can used in drying foods, such as salt and metabisulphite applications were carried out in drying tomatoes (Pazır et al., 1996). These are systems where there is not even an operating environment in direct sun drying or low investment and energy costs in greenhouse, tunnel, etc. systems. (Hnin et, al., 2018; Jin et al., 2017).

It is necessary to investigate the difference in terms of changes and differences in nutrients according to various drying methods, as well as in terms of basic food processes and drying parameters, in terms of technology and engineering calculations. (Akpınar, 2010; Li et al., 2020).

Lycopene, one of the most important compounds of beta carotenes, has functional properties such as; antioxidant effect (Oliveira et al., 2016).

This is one of the aims of this current research. The use of various drying methods increases the energy efficiency and ensures the production of quality products.

Traditional drying systems such as sun drying, but depending on time, season and weather conditions, food safety, non-technological, burning danger, environmental negative factors; Contamination by insects, rodents, other creatures, and even cat, dog and human contamination occurs. In this case, it becomes impossible to apply food safety standards. Adverse weather conditions, such as rain, lead to product losses.

Drying in the greenhouse is established in the project. Both types of drying are carried out in the greenhouse and in direct sunlight.

Although there are similar studies on these issues, there are almost no publications that examine both nutrients in some products, food safety and basic drying parameters together.

The quality of dried products is dependent on type of dryer and the drying conditions also biochemical composition and physical properties of the foods ( $\mathrm{Li}$ et al., 2020).

Dried foods products contain phytochemicals, such as vitamins, minerals, antioxidants, pigments and other bioactive compounds, associated with health benefits. It is well known that these components explore to significant degradation, losings, reductions o during drying, as they are sensitive to heat, light and oxygen (Nguyen and Schwartz, 1999).

Tomatoes with 90-98\% all-trans-lycopene as the main carotenoid component (Nguyen and Schwartz, 1999). Authors reported indicate that in contrast to $\beta$-carotene, lycopene remained relatively resistant to heat-induced geometrical conversion during typical food processing of tomatoes and related products (Minhthy and Steven, 1999). All-trans-lycopene was expose to degradation at temperatures above $70^{\circ} \mathrm{C}$ and lycopene bioavailability due to generated cis-isomers (Mayer-Miebach et al., 2005).

One of the aims of this study was to evaluate the combined effect of cultivation methods (conventional and two types of organic farming) and two different small-scale drying techniques on two tomato and bell pepper varieties) and similar study was present in the literature too (Paolo et al., 2019).
Mycotoxins are critical in terms of food safety. Ochratoxin levels have been investigated in major export food products of Manisa, and they were not found to be risky or dangerous in terms of food safety (Çăglarirmak, 2006). Aflatoxins can be formed in the form of harvest, drying, storage, food and feed, as well as in the field (Çoksöyler, 1999).

The minerals of the food products based on the determination of calcium, potassium, magnesium, phosphorus, sodium, zinc and iron. The researchers indicated that drying foods had no significant effect on the mineral elements (Eim et al., 2013).

Fruit and vegetables have functional provide useful positive effects to health such as properties and the protection of many chronic diseases, including cardiovascular disease, type II diabetes, dementia, macular degeneration and some cancers (Barrett and Lloyd, 2012).

The moisture contents of some dried products should be brought to the following ratios; meat, fish, and dairy $3 \%$ or less, vegetable products usually to $5 \%$, and cereal products $12 \%$ (Rahman and Perera, 2004).

Water activity values were ranging from 0.90 to 1.00 . In dehydration the highest water activity value must be around 0.60 for safe storage of vegetables. (Kaur et al., 2008).

Air-drying characteristics of tomatoes were investigated that found result was the increasing in the air temperature in the range $55-70^{\circ} \mathrm{C}$. markedly increased the drying rate of tomatoes (Doymaz, 2007).

\section{Materials and Methods}

\section{Materials}

Research samples: tomatoes, bell peppers, figs and graps were obtained in two different consecutive harvesting? years (2020-2021) Tomatoes and bell peppers were dried under the sun and into green house. Organic and natural tomatoes and bell peppers were dried in two different ways. In the research figs and grapes were dried under the sun. Grapes were sultanas dried by treating with alkaline solution with vegetable oil called as "potasa", the without treating without any solution, known as raisin.

Drying methods for tomatoes

X-Tomatoes organic, dried in the greenhouse, (TOG) I. Harvest

1.a-Tomatoes natural, dried under the sun, (TNS1)

1.b-Tomatoes natural, dried in the green house (TNG1) II. Harvest

11.a-Tomatoes natural, dried under the sun, (TNS2)

1l.b-Tomatoes natural, dried in the green house (TNG2)

Drying methods for bell peppers

y.Bell pepper natural, dried under the sun (PNS)

1. Harvest

1.a-Bell pepper organic dried under the sun (POS1)

1.b-Bell pepper organic dried in the green house (PNG1)

\section{Harvest}

11.a-Bell pepper organic dried under the sun (POS2)

11.b-Bell pepper organic dried in the green house (PNG2) 


\section{Drying methods for grapes and figs}

G. Grapes (sultanas) treating with potasa or alkali solution and vegetable oil,

R. Raisins, without treating any solution.

Sultanas and raisins were dried in the green house.

F. Figs organic, dried in the green house

In the research, depending on the material drying systems, the products were manufactured by drying under the sun or in the greenhouse. The experiments were carried out in a nylon greenhouse set up in the garden of the college. Drying in direct sunlight was also carried out on plastic shallow sieves. With a fan placed on the greenhouse wall inside the greenhouse, both the air flow inside the greenhouse was provided and the humid air formed was removed from here. Approximately 1-2 m/sec air velocity has been provided in the greenhouse.

\section{Methods}

Investigated main nutrients of dried samples were as follows; lycopene (in tomato samples), A, (in sultana and un-dipped (raisin) samples), vitamins; ascorbic acid (vitamin C), thiamine (B1), riboflavin (B2), pyridoxine (B6), retinoic acid (vitamin A), folic acid, copper $(\mathrm{Cu})$, iron $(\mathrm{Fe})$, zinc $(\mathrm{Zn})$, magnesium $(\mathrm{Mg})$, calcium $(\mathrm{Ca})$, sodium $(\mathrm{Na})$, potassium $(\mathrm{K})$, and contaminants; aflatoxin in red pepper and fig, ochratoxin.

In the study, same food components of dried tomatoes organic and natural, dried bell pepper were organic and natural were investigated in two different harvest periods.

Lycopene and vitamins were determined following references and methods: Lycopene; Zakaria et al., 1979, the vitamins were made according to these methods: B1, B2, B6 (19-23). (Finglas and Foulk, 1984; Kamman et al., 1980; A vitamin, Manz and Philiph, 1998; AOAC, 2000; folic acid R-Biopharm Art. No. 1002, AOAC, 2005; 999,985.35). Mineral analysis was carried out according to the AOAC standards (1990). (Çağlarırmak, 2007; Gökmen et al., 2000).
Ash was dissolved in $5 \mathrm{ml} \mathrm{20 \%} \mathrm{HCI,} \mathrm{diluted} \mathrm{and}$ filtered through $0.45 \mu \mathrm{m}$ pore size filter. Lanthanum was added to overcome interferences for $\mathrm{Ca}$ and $\mathrm{Mg}$ determination. Minerals were established by AAS, (atomic absorption spectrophotometer) except for $\mathrm{Na}$, K which were detected by FES (flame emission spectrophotometer) (AOAC, 2005; Çağlarırmak and Hepçimen, 2013).

Ochratoxin A (in raisins, dipped and not dipped samples were analyzed according to Rhone Diagnostics Technologies (1999) Notes (sultanas and raisin). Aflatoxin (total aflatoxin and aflatoxin B1 analysis was analyzed according to AOAC 2005 (999.07) (paprika and figs).

Moisture determinations and water activity analysis (aw): Moisture determinations were made with Sartorius automatic moisture meter scales. Water activity measurements were made with a Testo hand-held measuring device.

Statistical analysis was made according to Alpar, 2020.

\section{Results and Discussions}

In the research, Nutrients of sample and main mycotoxins in kinds of dryin methods were compared and applied statistic analysis were established in detail in the tables as follows:

Test was independent $t$ test. (Test 3). Tests whether there is a statistically significant difference between the mean of two independent groups.

When Table 1 is examined, there was significant differences at the level of $5 \%$ between the nutrients dried in the sun and in the greenhouse, except $\mathrm{B} 6, \mathrm{Zn}$ and $\mathrm{Cu}$.

There is a statistically significant difference between lcp, A vitamin, FA, C at the level of 5\%. However, there is no statistically significant difference between B1 and B6. (Table 5).

Lycopene amounts were investigated in the literature by Giovanelli et al., 2002) ( $\mathrm{mg} / \mathrm{kg} \mathrm{dm}$ ). Investigated lycopene values were in a large scale because of each of dried samples had different dry weights. Thus, the values found are close to the literature.

Table 1. Descriptive Statistics of the Nutrients of Greenhouse Dried Organic Tomato (TOG) (on Dry Weight Basis $\mathrm{mg} / 100 \mathrm{~g}, \mathrm{DWB} * *)$

\begin{tabular}{|c|c|c|c|c|c|c|}
\hline & \multicolumn{6}{|c|}{ Descriptive Statistics } \\
\hline & $\mathrm{N}$ & Min & Max & Mean & Std.Dev & Variance \\
\hline Lcp & 3 & 28.93 & 31.05 & 29.99 & 1.06 & 1.124 \\
\hline Avit ${ }^{*}$ & 3 & 14.64 & 15.70 & 15.17 & 0.53 & 0.281 \\
\hline B1 & 3 & 0.26 & 0.70 & 0.48 & 0.22 & 0.048 \\
\hline B2 & 3 & 1.15 & 2.67 & 1.91 & 0.76 & 0.578 \\
\hline B6 & 3 & 0.89 & 1.73 & 1.31 & 00.42 & 0.176 \\
\hline $\mathrm{FA}^{*}$ & 3 & 32.77 & 34.65 & 33.71 & 0.94 & 0.884 \\
\hline Cvit & 3 & 7.31 & 9.17 & 8.24 & 0.93 & 0.865 \\
\hline $\mathrm{Mg}$ & 3 & 130.89 & 132.51 & 131.7 & 0.81 & 0.656 \\
\hline $\mathrm{Zn}$ & 3 & 5.85 & 7.33 & 6.59 & 0.74 & 0.548 \\
\hline $\mathrm{Ca}$ & 3 & 94.02 & 94.98 & 94.5 & 0.48 & 0.230 \\
\hline $\mathrm{Na}$ & 3 & 11429.71 & 11430.29 & 11430 & 0.29 & 0.084 \\
\hline $\mathrm{K}$ & 3 & 2620.53 & 2622.47 & 2621.5 & 0.97 & 0.941 \\
\hline $\mathrm{Fe}$ & 3 & 6.16 & 7.58 & 6.87 & 0.71 & 0.504 \\
\hline $\mathrm{Cu}$ & 3 & 0.56 & 0.58 & 0.57 & 0.01 & 0.000 \\
\hline $\mathrm{P}$ & 3 & 177.41 & 177.79 & 177.6 & 0.19 & 0.036 \\
\hline Valid N & 3 & & & & & \\
\hline
\end{tabular}


Table 2. Descriptive Statistics on Nutritional Values of Greenhouse and Sun-dried Natural Tomatoes in 1st Harvest Time (on Dry Weight Basis mg/100g, dw**)

\begin{tabular}{|c|c|c|c|c|c|}
\hline & \multicolumn{5}{|c|}{ Group Statistics } \\
\hline & TNG1/TNS1 & $\mathrm{N}$ & Mean & Std. Deviation & Std. Error Mean \\
\hline \multirow{2}{*}{ Lcp } & Natural Greenhouse & 3 & 18.4800 & 1.25000 & 0.72169 \\
\hline & Natural Undersun & 3 & 51.7000 & 1.38000 & 0.79674 \\
\hline \multirow{2}{*}{ Avit $^{*}$} & Natural Greenhouse & 3 & 12.8900 & 0.56000 & 0.32332 \\
\hline & Natural Undersun & 3 & 20.6900 & 0.98000 & 0.56580 \\
\hline \multirow{2}{*}{ B6 } & Natural Greenhouse & 3 & 0.7300 & 0.28000 & 0.16166 \\
\hline & Natural Undersun & 3 & 0.7700 & 0.70000 & 0.40415 \\
\hline \multirow{2}{*}{$\mathrm{FA}^{*}$} & Natural Greenhouse & 3 & 22.9800 & 0.07000 & 0.04041 \\
\hline & Natural Undersun & 3 & 243.3000 & 0.17000 & 0.09815 \\
\hline \multirow{2}{*}{ Cvit } & Natural Greenhouse & 3 & 5.8400 & 0.34000 & 0.19630 \\
\hline & Natural Undersun & 3 & 9.1900 & 0.16000 & 0.09238 \\
\hline \multirow{2}{*}{$\mathrm{Mg}$} & Natural Greenhouse & 3 & 106.7000 & 0.79000 & 0.45611 \\
\hline & Natural Undersun & 3 & 155.6000 & 0.52000 & 0.30022 \\
\hline \multirow{2}{*}{$\mathrm{Zn}$} & Natural Greenhouse & 3 & 3.5800 & 0.53000 & 0.30600 \\
\hline & Natural Undersun & 3 & 2.8400 & 0.14000 & 0.08083 \\
\hline \multirow{2}{*}{$\mathrm{Ca}$} & Natural Greenhouse & 3 & 85.4000 & 0.56000 & 0.32332 \\
\hline & Natural Undersun & 3 & 90.9000 & 0.08000 & 0.04619 \\
\hline \multirow{2}{*}{$\mathrm{Na}$} & Natural Greenhouse & 3 & 8499.5000 & 0.40000 & 0.23094 \\
\hline & Natural Undersun & 3 & 8477.0000 & 0.74000 & 0.42724 \\
\hline \multirow{2}{*}{ K } & Natural Greenhouse & 3 & 2486.0000 & 0.07000 & 0.04041 \\
\hline & Natural Undersun & 3 & 3275.0000 & 0.86000 & 0.49652 \\
\hline \multirow{2}{*}{$\mathrm{Fe}$} & Natural Greenhouse & 3 & 6.6500 & 0.62000 & 0.35796 \\
\hline & Natural Undersun & 3 & 4.6800 & 0.22000 & 0.12702 \\
\hline \multirow{2}{*}{$\mathrm{Cu}$} & Natural Greenhouse & 3 & 00.6300 & 0.05000 & 0.02887 \\
\hline & Natural Undersun & 3 & 0.6533 & 0.02517 & 0.01453 \\
\hline \multirow{2}{*}{$\mathrm{P}$} & Natural Greenhouse & 3 & 167.9000 & 0.49000 & 0.28290 \\
\hline & Natural Undersun & 3 & 228.8000 & 0.80000 & 0.46188 \\
\hline
\end{tabular}

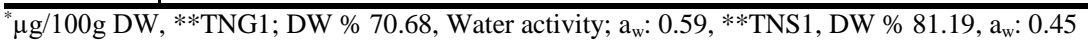

Table 3. The Results of Independent Samples t test on Nutritional Values of Greenhouse and Sun-dried Natural Tomatoes in 1st Harvest Time (on Dry Weight Basis mg/100g, dw)

\begin{tabular}{|c|c|c|c|c|c|c|}
\hline & & \multicolumn{5}{|c|}{ Independent Samples Test } \\
\hline & & \multicolumn{3}{|c|}{$\begin{array}{c}\text { Levene's Test for Equality of } \\
\text { Variances }\end{array}$} & \multicolumn{2}{|c|}{ t-test for Equality of Means } \\
\hline & & $\mathrm{F}$ & Sig. & $\mathrm{T}$ & Df & Sig. (2-tailed) \\
\hline Lcp & Equal variances assumed & 0.019 & 0.896 & -30.902 & 4 & 0.000 \\
\hline Avit* & Equal variances assumed & 0.554 & 0.498 & -11.969 & 4 & 0.000 \\
\hline B6 & Equal variances assumed & 1.241 & 0.328 & -.092 & 4 & 0.931 \\
\hline $\mathrm{FA}^{*}$ & Equal variances assumed & 1.183 & 0.338 & -2075.660 & 4 & 0.000 \\
\hline Cvit & Equal variances assumed & 0.918 & 0.392 & -15.441 & 4 & 0.000 \\
\hline $\mathrm{Mg}$ & Equal variances assumed & 0.326 & 0.599 & -89.553 & 4 & 0.000 \\
\hline $\mathrm{Zn}$ & Equal variances assumed & 2.025 & 0.228 & 2.338 & 4 & 0.080 \\
\hline $\mathrm{Ca}$ & Equal variances assumed & 2.880 & 0.165 & -16.840 & 4 & 0.000 \\
\hline $\mathrm{Na}$ & Equal variances assumed & 0.653 & 0.464 & 46.329 & 4 & 0.000 \\
\hline $\mathrm{K}$ & Equal variances assumed & 3.353 & 0.141 & -1583.818 & 4 & 0.000 \\
\hline $\mathrm{Fe}$ & Equal variances assumed & 1.479 & 0.291 & 5.187 & 4 & 0.007 \\
\hline $\mathrm{Cu}$ & Equal variances assumed & 0.731 & 0.441 & -.722 & 4 & 0.510 \\
\hline $\mathrm{P}$ & Equal variances assumed & 0.437 & 0.545 & -112.438 & 4 & 0.000 \\
\hline
\end{tabular}

The amount of lycopene contained in the samples within a certain range also varies according to the amount of DM, but it is within acceptable limits. In addition to its powerful antioxidant properties, lycopene becomes more active in heat-treated foods, so consumption of dry tomatoes such as 50-100 $\mathrm{g}$ as a direct table food can be protective and preventive, especially in preventing prostate cancer or the growth of the prostate gland and even the formation of other types of cancer (Willis and Wiani, 2003).
Biochemical compositions or nutrients dried tomatoes were given Tables 1, 2. On the other hand, Descriptive Statistics on Nutritional values and independent sample tests were shown in Table, 3, 4, 5.

Since B1 thiamine deficiency, the disease occurs and is one of the coenzymes (Sencer, 1983). It plays an important role in energy metabolism in the body. Thiamine values in the study in mg / $100 \mathrm{~g}$ on a DM basis in dry tomatoes (0.48-2.53). 
Table 4. Descriptive Statistics on Nutritional Values of Greenhouse and Sun-dried Natural Tomatoes in 2nd Harvest Time (on Dry Weight Basis mg/100g, DWB**)

\begin{tabular}{|c|c|c|c|c|c|}
\hline & \multicolumn{5}{|c|}{ Group Statistics } \\
\hline & TNG1/TNS2 & $\mathrm{N}$ & Mean & Std. Deviation & Std. Error Mean \\
\hline \multirow{2}{*}{ Lcp } & Natural Greenhouse & 3 & 44.27 & 1.02 & 0.59 \\
\hline & Natural Undersun & 3 & 49.33 & 0.035 & 0.02 \\
\hline \multirow{2}{*}{ Avit ${ }^{*}$} & Natural Greenhouse & 3 & 25.19 & 0.575 & 0.33 \\
\hline & Natural Undersun & 3 & 16.58 & 0.07 & 0.04 \\
\hline \multirow{2}{*}{ B1 } & Natural Greenhouse & 3 & 0.30 & 0.01 & 0.0058 \\
\hline & Natural Undersun & 3 & 0.31 & 0.01 & 0.0058 \\
\hline \multirow{2}{*}{ B2 } & Natural Greenhouse & 3 & 0.02 & $0.00000^{\mathrm{a}}$ & 0.00000 \\
\hline & Natural Undersun & 3 & 0.03 & $0.00000^{\mathrm{a}}$ & 0.00000 \\
\hline \multirow{2}{*}{ B6 } & Natural Greenhouse & 3 & 0.12 & 0.01 & 0.0058 \\
\hline & Natural Undersun & 3 & 0.11 & 0.0058 & 0.003 \\
\hline \multirow{2}{*}{$\mathrm{FA}^{*}$} & Natural Greenhouse & 3 & 15.41 & 0.065 & 0.0378 \\
\hline & Natural Undersun & 3 & 82.2 & 1 & 0.58 \\
\hline \multirow{2}{*}{ Cvit } & Natural Greenhouse & 3 & 18.06 & 0.14 & 0.081 \\
\hline & Natural Undersun & 3 & 21.13 & 0.78 & 0.45 \\
\hline
\end{tabular}

Table 5. The Results of Independent Samples t test on Nutritional Values of Greenhouse and Sun-dried Natural Tomatoes in 2nd Harvest Time (on Dry Weight Basis mg/100g, dw)

\begin{tabular}{|c|c|c|c|c|c|c|}
\hline & & \multicolumn{5}{|c|}{ Independent Samples Test } \\
\hline & & \multicolumn{2}{|c|}{$\begin{array}{c}\text { Levene's Test for Equality } \\
\text { of Variances }\end{array}$} & \multicolumn{3}{|c|}{ t-test for Equality of Means } \\
\hline & & $\mathrm{F}$ & Sig. & $\mathrm{T}$ & Df & Sig. (2-tailed) \\
\hline Lcp & Equal variances assumed & 3.714 & 0.126 & -8.582 & 4 & 0.001 \\
\hline Avit* & Equal variances assumed & 3.096 & 0.153 & 25.735 & 4 & 0.000 \\
\hline \multirow[t]{2}{*}{ B1 } & Equal variances assumed & 0.000 & 1.000 & -1.225 & 4 & 0.288 \\
\hline & Equal variances not assumed & & & -1.225 & 4.000 & 0.288 \\
\hline B6 & Equal variances assumed & 0.400 & 0.561 & 1.000 & 4 & 0.374 \\
\hline $\mathrm{FA}^{*}$ & Equal variances assumed & 3.471 & 0.136 & -115.445 & 4 & 0.000 \\
\hline Cvit & Equal variances assumed & 2.609 & 0.182 & -6.710 & 4 & 0.003 \\
\hline
\end{tabular}

B2 riboflavin was studied in the same examples. Riboflavin is a light-sensitive vitamin and together with niacin, which acts as a coenzyme, it is a vitamin that causes Pellagra disease in its deficiency. Dry weight or dry material, $\mathrm{mg} / 100 \mathrm{~g}$ in dried tomatoes (0.81-1.92).

The values found show that dried tomato samples can contribute to nutrition in terms of riboflavin. Vitamin A retinol amounts were determined. Vitamin $\mathrm{A}$ is a fatsoluble vitamin and shows antioxidant properties. According to this, vitamin A values in dry tomato samples are $\mu \mathrm{g} / 100 \mathrm{~g}$ on DM basis; It was detected in the range of (12.89-20.69) (Table 2).

Pyridoxine (B6) Dry Matter mg / 100 in dried tomato samples; (0.73-1.31). In dried tomato samples (Ascorbic acid), vitamin C was found as DW (mg / $100 \mathrm{~g}$ ) (5.849.19).

Vitamin C (Ascorbic acid) KM (mg / $100 \mathrm{~g}$ ) in dried tomato samples (5.84-9.19). It was the so sensitive vitamin against physical and chemical factors. Dried tomato samples cannot provide whole vitamin $\mathrm{C}$ requirement of body.

Folic acid (FA) is one of the important group B vitamins and megaloblastic anemia occurs in its deficiency (Sencer, 83). Taking pregnancy in the first months of pregnancy is important for the healthy course of pregnancy for the good development of the fetus. FA was detected in the samples at the level of $\mu \mathrm{g} / 1000 \mathrm{~g}$.

Vitamins are sensitive nutrients that can be affected quickly and easily by physical factors such as heat, light, oxygen, heavy metals, acidic environment or alkaline environment, $\mathrm{pH}$. Exposure to heat, light and oxygen are important factors in research. All samples were sliced or divided and dried in the drying greenhouse set up in the garden of the school. Changes in dry matter ratios in the samples affected the vitamin quantities.

Minerals are cofactors and are important nutrients that the body needs. In a balanced diet, it should be evaluated within important food groups as well as other nutrients (Table1,2).

Minerals may increase in quantity on DM basis in dried products. This is an expected situation.

However, there may be changes in the quantities of salting mineral substances such as salt $(\mathrm{NaCl})$, sulfur dioxide, or other preservative or other additives, contamination from drying vessels or as expected additives.

Magnesium $(\mathrm{Mg})$ plays a role in the regulation of intestinal peristaltic movements. It is found in the cardiovascular system and bone structure. In this current research, $\mathrm{Mg}$ in dried tomatoes was detected in the range of $\mathrm{mg} / 100 \mathrm{~g}$ (131.7-155.55) in dry tomatoes. 
Potassium $(\mathrm{K})$ is the important electrolyte found in intracellular fluid. Dried tomatoes showed a wide range value of DW mg / $100 \mathrm{~g}$ (2486-32750). Approximately $90 \%$ increase in dry matter basis caused these values to increase. The consumption of small amounts of dried vegetable samples meets the $\mathrm{K}$ requirement, but consumption of these products may not be recommended even as 50-100 $\mathrm{g}$ for chronic kidney patients.
Sodium (Na) is an important electrolyte found in the extracellular fluid, while others have increased the DM basis, as well as increasing the $\mathrm{Na}$ quantities of salt-dried products. It shows range values around tenfold. Dried tomatoes contained $\mathrm{Na}$ in range $\mathrm{mg} / 100 \mathrm{~g}$ in dry weight basis (1143-84995).

Table 6. Descriptive Statistics of the Nutrients of Greenhouse Dried Naturel Bell Pepper (PNS) (on Dry Weight Basis $\mathrm{mg} / 100 \mathrm{~g}, \mathrm{DWB} * *)$

\begin{tabular}{|c|c|c|c|c|c|c|}
\hline & \multicolumn{6}{|c|}{ Descriptive Statistics } \\
\hline & $\mathrm{N}$ & Min & Max & Mean & Std. Dev & Variance \\
\hline Avit $^{*}$ & 3 & 39.50 & 40.86 & 40.18 & 0.68 & 0.462 \\
\hline B1 & 3 & 1.32 & 2.34 & 1.83 & 0.51 & 0.260 \\
\hline B6 & 3 & 0.23 & 1.25 & 0.74 & 0.51 & 0.260 \\
\hline $\mathrm{FA}^{*}$ & 3 & 201.28 & 201.72 & 201.5 & 0.22 & 0.048 \\
\hline Cvit & 3 & 440.78 & 441.30 & 441.04 & 0.26 & 0.068 \\
\hline $\mathrm{Mg}$ & 3 & 152.23 & 152.77 & 152.5 & 0.27 & 0.073 \\
\hline $\mathrm{Zn}$ & 3 & 5.16 & 5.64 & 5.4 & 0.24 & 0.058 \\
\hline $\mathrm{Ca}$ & 3 & 47.84 & 49.36 & 48.6 & 0.76 & 0.578 \\
\hline $\mathrm{Na}$ & 3 & 470.10 & 471.10 & 470.6 & 0.50 & 0.250 \\
\hline $\mathrm{K}$ & 3 & 2794.88 & 2796.12 & 2795.5 & 0.62 & 0.384 \\
\hline $\mathrm{Fe}$ & 3 & 14.10 & 14.70 & 14.4 & 0.30 & 0.090 \\
\hline $\mathrm{Cu}$ & 3 & 0.31 & 1.49 & 0.9 & 0.59 & 0.348 \\
\hline $\mathrm{P}$ & 3 & 266.82 & 266.98 & 266.9 & 0.08 & 0.006 \\
\hline Valid N (listwise) & 3 & & & & & \\
\hline
\end{tabular}

* $\mu \mathrm{g} / 100 \mathrm{~g}$ DW, (PNS) DWB \% 86.87 aw $=0.40$

Table 7. Descriptive Statistics on Nutritional Values of Greenhouse and Sun-dried Organic Bell Pepper in (POS1) 1st Harvest Time (on Dry Weight Basis mg/100g, dw)

\begin{tabular}{|c|c|c|c|c|c|}
\hline & \multicolumn{5}{|c|}{ Group Statistics } \\
\hline & POS1/PNG1 & $\mathrm{N}$ & Mean & Std. Deviation & Std. Error Mean \\
\hline \multirow{2}{*}{ Avit* } & Organic Greenhouse & 3 & 25.0500 & 0.76000 & 0.43879 \\
\hline & Organic Undersun & 3 & 23.0100 & 0.95000 & 0.54848 \\
\hline \multirow{2}{*}{ B1 } & Organic Greenhouse & 3 & 1.8700 & 0.75000 & 0.43301 \\
\hline & Organic Undersun & 3 & 1.3400 & 0.70171 & 0.40513 \\
\hline \multirow{2}{*}{ B2 } & Organic Greenhouse & 3 & 0.4400 & 0.10000 & 0.05774 \\
\hline & Organic Undersun & 3 & 0.3800 & 0.02000 & 0.01155 \\
\hline \multirow{2}{*}{ B6 } & Organic Greenhouse & 3 & 1.0800 & 0.10000 & 0.05774 \\
\hline & Organic Undersun & 3 & .6133 & 0.11015 & 0.06360 \\
\hline \multirow{2}{*}{$\mathrm{FA}^{*}$} & Organic Greenhouse & 3 & 179.2500 & 0.28000 & 0.16166 \\
\hline & Organic Undersun & 3 & 207.8000 & 0.78000 & 0.45033 \\
\hline \multirow{2}{*}{ Cvit } & Organic Greenhouse & 3 & 10.6500 & 0.92000 & 0.53116 \\
\hline & Organic Undersun & 3 & 3.3900 & 0.31000 & 0.17898 \\
\hline \multirow{2}{*}{$\mathrm{Mg}$} & Organic Greenhouse & 3 & 140.1000 & 0.52000 & 0.30022 \\
\hline & Organic Undersun & 3 & 143.8000 & 0.16000 & 0.09238 \\
\hline \multirow{2}{*}{$\mathrm{Zn}$} & Organic Greenhouse & 3 & 3.3500 & 0.39000 & 0.22517 \\
\hline & Organic Undersun & 3 & 9.3600 & 0.06000 & 0.03464 \\
\hline \multirow{2}{*}{$\mathrm{Ca}$} & Organic Greenhouse & 3 & 52.3000 & 0.20000 & 0.11547 \\
\hline & Organic Undersun & 3 & 61.7000 & 0.93000 & 0.53694 \\
\hline \multirow{2}{*}{$\mathrm{Na}$} & Organic Greenhouse & 3 & 366.2000 & 0.08000 & 0.04619 \\
\hline & Organic Undersun & 3 & 3526.5000 & 0.74000 & 0.42724 \\
\hline \multirow{2}{*}{ K } & Organic Greenhouse & 3 & 2846.0000 & 0.30000 & 0.17321 \\
\hline & Organic Undersun & 3 & 2846.5000 & 0.84000 & 0.48497 \\
\hline \multirow{2}{*}{$\mathrm{Fe}$} & Organic Greenhouse & 3 & 4.1000 & 0.66000 & 0.38105 \\
\hline & Organic Undersun & 3 & 8.9000 & 0.56000 & 0.32332 \\
\hline \multirow{2}{*}{$\mathrm{Cu}$} & Organic Greenhouse & 3 & 1.0200 & 0.99000 & 0.57158 \\
\hline & Organic Undersun & 3 & 0.7400 & 0.14000 & 0.08083 \\
\hline \multirow{2}{*}{$\mathrm{P}$} & Organic Greenhouse & 3 & 279.2000 & 0.44000 & 0.25403 \\
\hline & Organic Undersun & 3 & 310.3000 & 0.37000 & 0.21362 \\
\hline
\end{tabular}

* $\mu \mathrm{g} / 100 \mathrm{~g}$ DW, (POS1) DWB \% 84.84 aw: 0.41 
Table 8. The Results of Independent Samples t test on Nutritional Values of Greenhouse and Sun-dried Organic Bell

\begin{tabular}{|c|c|c|c|c|c|c|}
\hline & & \multicolumn{5}{|c|}{ Independent Samples Test } \\
\hline & & \multicolumn{2}{|c|}{ Levene's Test for Equality of Variances } & \multicolumn{3}{|c|}{ t-test for Equality of Means } \\
\hline & & $\mathrm{F}$ & Sig. & $\mathrm{t}$ & df & Sig. (2-tailed) \\
\hline Avit & Equal variances assumed & 0.098 & 0.770 & 2.904 & 4 & 0.044 \\
\hline B1 & Equal variances assumed & 0.004 & 0.950 & 0.894 & 4 & 0.422 \\
\hline B2 & Equal variances assumed & 2.462 & 0.192 & 1.019 & 4 & 0.366 \\
\hline B6 & Equal variances assumed & 0.034 & 0.862 & 5.433 & 4 & 0.006 \\
\hline FA & Equal variances assumed & 1.456 & 0.294 & -59.669 & 4 & 0.000 \\
\hline Cvit & Equal variances assumed & 1.579 & 0.277 & 12.953 & 4 & 0.000 \\
\hline $\mathrm{Mg}$ & Equal variances assumed & 1.751 & 0.256 & -11.779 & 4 & 0.000 \\
\hline $\mathrm{Zn}$ & Equal variances assumed & 2.798 & 0.170 & -26.381 & 4 & 0.000 \\
\hline $\mathrm{Ca}$ & Equal variances assumed & 2.356 & 0.200 & -17.115 & 4 & 0.000 \\
\hline $\mathrm{Na}$ & Equal variances assumed & 3.145 & 0.151 & -7354.177 & 4 & 0.000 \\
\hline K & Equal variances assumed & 1.466 & 0.293 & -0.971 & 4 & 0.387 \\
\hline $\mathrm{Fe}$ & Equal variances assumed & 0.053 & 0.829 & -9.605 & 4 & 0.001 \\
\hline $\mathrm{Cu}$ & Equal variances assumed & 2.891 & 0.164 & 0.485 & 4 & 0.653 \\
\hline $\mathrm{P}$ & Equal variances assumed & 0.059 & 0.820 & -93.699 & 4 & 0.000 \\
\hline
\end{tabular}

* $\mu \mathrm{g} / 100 \mathrm{~g}$ DW, (PNG1) DWB: \% 84.02, $\mathrm{a}_{\mathrm{w}}: 0.45$

Table 9. Descriptive Statistics on Nutritional Values of Greenhouse and Sun-dried Organic Bell Pepper (POS2) in 2nd Harvest Time (on Dry Weight Basis mg/100g, dw)

\begin{tabular}{l|llccc}
\hline \multirow{2}{*}{ Avit } & \multicolumn{4}{c}{ Group Statistics } \\
\cline { 2 - 6 } & \multicolumn{1}{c}{ POS2/PNG2 } & $\mathrm{N}$ & Mean & Std. Deviation & Std. Error Mean \\
\hline \multirow{3}{*}{ B1 } & Organic Greenhouse & 3 & 7.0167 & 0.02517 & 0.01453 \\
& Organic Undersun & 3 & 7.4500 & 0.20000 & 0.11547 \\
B2 & Organic Greenhouse & 3 & 0.8567 & 0.03512 & 0.02028 \\
& Organic Undersun & 3 & 0.9467 & 0.01528 & 0.00882 \\
B6 & Organic Greenhouse & 3 & 0.3300 & 0.01000 & 0.00577 \\
& Organic Undersun & 3 & 0.4900 & 0.00000 & 0.00000 \\
FA & Organic Greenhouse & 3 & 0.4067 & 0.00577 & 0.00333 \\
& Organic Undersun & 3 & 1.2400 & 0.01000 & 0.00577 \\
Cvit & Organic Greenhouse & 3 & 176.5500 & 1.95000 & 1.12583 \\
& Organic Undersun & 3 & 197.1000 & 3.30000 & 1.90526 \\
\hline
\end{tabular}

(POS2) DWB: $84.5 \% \mathrm{a}_{\mathrm{w}} 0.37$

Table 10. The Results of Independent Samples t test on Nutritional Values of Greenhouse and Sun-dried Organic Bell Pepper (PNG2) in 2nd Harvest Time (on Dry Weight Basis mg/100g, dw)

\begin{tabular}{|c|c|c|c|c|c|c|}
\hline & & \multicolumn{5}{|c|}{ Independent Samples Test } \\
\hline & & \multicolumn{2}{|c|}{$\begin{array}{l}\text { Levene's Test for Equality of } \\
\text { Variances }\end{array}$} & \multicolumn{3}{|c|}{ t-test for Equality of Means } \\
\hline & & $\mathrm{F}$ & Sig. & $\mathrm{t}$ & Df & Sig. (2-tailed) \\
\hline Avit & Equal variances assumed & 2.969 & 0.160 & -3.723 & 4 & 0.020 \\
\hline B1 & Equal variances assumed & 1.385 & 0.305 & -4.070 & 4 & 0.015 \\
\hline B2 & Equal variances assumed & 4.000 & 0.116 & -27.713 & 4 & 0.000 \\
\hline B6 & Equal variances assumed & 0.400 & 0.561 & -125.000 & 4 & 0.000 \\
\hline FA & Equal variances assumed & 0.496 & 0.520 & -9.286 & 4 & 0.001 \\
\hline Cvit & Equal variances assumed & 3.443 & 0.137 & -161.690 & 4 & 0.000 \\
\hline
\end{tabular}

(PNG2) DWB: 85.14\% $\mathrm{a}_{\mathrm{w}}: 0.45$

High levels of both $\mathrm{Na}$ and $\mathrm{K}$ in products are not a good rate in terms of cardiovascular diseases. Products with low $\mathrm{Na}$ content but high $\mathrm{K}$ content are beneficial in terms of cardiovascular diseases.

The quantities of minerals such as $\mathrm{Ca}, \mathrm{Mg}, \mathrm{Na}, \mathrm{K}$ were found to be high values in the research. In the products dried by sprinkling salt, the salt added as a homogeneous solution by sprinkling different randomly and drying has increased the values of $\mathrm{K}, \mathrm{Mg}$ and $\mathrm{Ca}$, especially $\mathrm{Na}$, which may come from the salt, compared to the literature (Demirbaş, 2010).

Zinc $(\mathrm{Zn})$ is one of the important cofactors and is a mineral that contributes positively to the epithelium tissue in wound healing. Zinc amounts in dry tomatoes were determined in the range of $\mathrm{mg} / 100 \mathrm{~g}$ (6.59-35.84) on dry matter basis. 
Iron $(\mathrm{Fe})$ is an essential trace element that carries oxygen to anemic tissues in Fe deficiency. Fe quantities in dried tomato samples were determined in the range of $\mathrm{mg}$ / 100g d (6.87-66.47) on dry matter weight basis.

Copper $(\mathrm{Cu})$ is the cofactor that causes copper deficiency anemia in the deficiency of large amounts in the body. It is in the structure of polyphenol oxidase enzyme in plants. This causes enzymatic browning reactions. Dry matter in dried tomato samples is in the range of $\mathrm{Cu} \mathrm{mg} /$ $100 \mathrm{~g}(0.56-6.50)$.

Descriptive Statistics and Independent Samples dried bell peppers Tests were given tables 6, 7, 8, 9, and 10. Each of chemical composition and nutrient contents were evaluated for nutrition impacts. In the literature very less studies were present and dried sample values were varied according to dry basis of tomatoes and peppers.

Vitamin B1 in chili peppers on a mg / 100g dry matter basis (1.14-1.87). It has been found in the range. In terms of thiamine, peppers show more stable and compatible values. In dried red peppers, B2 riboflavin vitamin on the basis of mg / $100 \mathrm{~g}$ dry matter weight; Vitamin A was found in the range of (0.38-0.48) and retinol in the range of (23.01-40.18) in red peppers. Vitamin A content of dried red peppers can contribute to nutrition in terms of vitamin A.

B6 pyridoxine values in dried peppers are $\mathrm{mg} / 100 \mathrm{~g}$ on dry matter weight basis; while it is in the range of $(0.41$ $1.25)$, it is surprising that vitamin $C$ is found in a very wide range in dry pepper samples, but the difference in the amount of dry matter may be due to such situations.

Folic acid FA was determined in dry red pepper samples in the range of $\mathrm{mg} / 100 \mathrm{~g}$ (179.25- 207.8) on dry matter weight basis. Especially k. It has been determined that red peppers are rich in folic acid and give the highest values. Essential nourishing minarets were found in the samples in the study.

Magnesium Mg in dried pepper samples, $\mathrm{mg} / 100 \mathrm{~g}$ on dry matter weight basis; (1401-1525) Potassium K found in intracellular fluid (27450-28465), and sodium $\mathrm{Na}$ in the cell decay fluid (3662-35265).

High levels of both $\mathrm{Na}$ and $\mathrm{K}$ in products are not a good rate in terms of cardiovascular diseases. Products with low $\mathrm{Na}$ content but high $\mathrm{K}$ content are beneficial in terms of cardiovascular diseases.

As mentioned before, the quantities of minerals such as $\mathrm{Ca}, \mathrm{Mg}, \mathrm{Na}, \mathrm{K}$ were found to be high values in the research. In the products dried by sprinkling salt, the salt added as a homogeneous solution by sprinkling different randomly and drying has increased the values of $\mathrm{K}, \mathrm{Mg}$ and $\mathrm{Ca}$, especially $\mathrm{Na}$, which may come from the salt, compared to the literature (Demirbaş, 2010).

Phosphorus $\mathrm{P}$ is one of the important essential nutrient minerals found in bones and teeth after calcium in energy metabolism. It is especially high in foods with high protein content.

The values found in the research examples (Table 2) show that these products can contribute significantly to nutrition.

In dried pepper samples, on dry matter weight basis $\mathrm{mg}$ / 100 Zinc Zn; (33.53-93.47) Iron Fe; (40.19- 143.7) and Copper $\mathrm{Cu}$; It has been determined as $(8,99-10,19)$ and $\mathrm{Cu}$ is at a level that can contribute to nutrition in research samples.

\author{
Mycotoxin Results in The Research \\ Aflatoxins \\ Pepper 8: Total aflatoxin (B1, B2, G1, G2): <0.5ng / g \\ Aflatoxin B1: $<0.2 \mathrm{ng} / \mathrm{g}$ \\ Pepper 9: Total aflatoxin (B1, B2, G1, G2): $<0.5 \mathrm{ng} / \mathrm{g}$ \\ Aflatoxin B1: $<0.2 \mathrm{ng} / \mathrm{g}$ \\ Pepper 13: Total aflatoxin (B1, B2, G1, G2): $<0.5 \mathrm{ng} / \mathrm{g}$ \\ Aflatoxin B1: $<0.2 \mathrm{ng} / \mathrm{g}$
}

\section{Ochratoxins OTA}

Grape dipped (sultanas): Ochratoxin A: $<0.23 \mathrm{ng} / \mathrm{g}$

Grape not dipped (raisin): Ochratoxin A: $1.04 \mathrm{ng} / \mathrm{g}$.

Dried fruits and vegetables are preferred because of their nutritional energy value together with addition of kinds of foods because of their contribution to both taste and nutritional value according fresh products (Debra et al., 2011).

Another aim of this research is to investigate products in terms of food safety as well as the research of nutrients for preparing food labels. Preserving food by drying is one of the oldest methods and has been the subject of national and international research in terms of both quality control and basic processes Çağlarırmak, 2006; Cemeroğlu and et al., 2009).

Aflatoxins found in food and feed are under severe control. The US Food and Drug Administration (FDA) has determined the maximum $20 \mathrm{ng} / \mathrm{g}$ that can be found in foodstuffs.

AFs are listed as AFB1 $>$ AFG2 $>$ afb2 $>$ afg2 according to the toxin potency they create. AFB1 has the highest toxic effect. However, in the EU regulation published for the last time on 8 March 2010, there was no change in the amount of AF B1 in dried fruits (Şen ve Nas, 2010). This limit has been determined as $4 \mathrm{ng} / \mathrm{g}$ for total AF and $2 \mathrm{ng} / \mathrm{g}$ for AFB1 as the European Union limits (Anonymous, 2007; Şen and Nas, 2010).

Aflatoxin (AF) and Ochratoxin (OTA) are important danger and risk factors that constitute critical control points in food processes that cause cancer-causing liver and kidney tumors. No method can ensure that the toxins are eliminated after these toxins are present in the products at a high rate. In this case, there is no other option but to destroy the product.

Total aflatoxin contents in dried red pepper products were analyzed. Total aflatoxin was found to be $\langle 0.5$, $>$ $0.5,>0.5$ for pepper 8 , pepper 9 and pepper 13 , and $>0.2$, 0.2 and 0.2 for aflatoxin B1. In figs, it was found as $0.1 \mathrm{ng}$ / g, $0.024 \mathrm{ng} / \mathrm{g}$, and considering that it is $4 \mathrm{ng} / \mathrm{g}$ for total aflatoxin and $2 \mathrm{ng} / \mathrm{g}$ for AFB1 as the European Union limits, aflatoxins found do not pose a risk or danger.

It is well below EU limits. OTA grape values are $<0.23$ $\mathrm{ng} / \mathrm{g}$ for sultanas and $1.04 \mathrm{ng} / \mathrm{g}$ for raisin. Considering that the EU limits are $10 \mu \mathrm{g} / \mathrm{kg}$, very low values are obtained. Grapes do not pose any danger or risk in terms of OTA.

Mycotoxin study is limited in this study. Because the healthy harvest of the products, their drying without contact with the soil and their short-term but cold storage have been important factors in obtaining low mycotoxin values. 


\section{Conclusion}

Dried and semi dried tomatoes and bell peppers were consumed in a large quantity together with grapes (sultanas and figs) also all of they are the important importing products of Turkey. There are sorts of drying processes such as under the sun, in greenhouse and solar drying. In the research there were the main drying systems; under the sun and greenhouse. The comparing data of nutrients were obtained between the two systems drying because of there are the affecting factors of nutrient values those were exposing the sun and oxygen, $\mathrm{pH}$, drying temperature and period. These nutrients were including minerals were investigated in dry weight or dry matter bases. They were explained in statically descriptive Statistics on Nutritional values and independent $t$ tests.

The main evidence of the research was studied nutrients in dried tomatoes and bell peppers can contribute the nutrition daily intake. The comparing data of nutrients in drying systems were varied and could explained statistically. Data were determined in different harvesting periods for dried tomatoes and peppers.

On the other hand, mycotoxins cause to food safety problems for health and in the market. Mycotoxin levels dried grapes and figs were determined and found that no hazardous levels of mycotoxins were found in the dried grapes and figs. They were safer from point of mycotoxin levels.

\section{Acknowledgement}

The authors are thankful to Celal Bayar University Scientific Research Project Commission for both their financial and moral support in Manisa City, TUB<TAKMAM (The Scientific and Technological Research Council of Turkey-Marmara Research Center) Food Research and Development Analysis laboratory of TUBITAK in Kocaeli City. We would also like to thank the following public and private sector organizations for their support; TÜBİTAK MAM Food Institute Laboratories, Saruhanlı Vocational School personnel who contributed to the project.

- We would like to thank especially the technical staff Mehmet Kayar, who is in our memories and lost suddenly. - Kybele Gourmet Ltd.,

- Şenaylar Petrol, Food Cotton Ltd.,

- Salkım Agricultural Products Ltd.

\section{References}

Akpinar EK. 2010. Drying of mint leaves in a solar dryer and under open sun: modelling, performance analyses. Energy conversion and management, 51(12), 2407-2418. https://doi.org/10.1016/j.enconman.2010.05.005

Alpar R. 2020. Uygulamalı istatistik ve geçerlilik güvenirlik, p. 282, Ankara. (in Turkish)

Anonymous 2007. EC Commission of Regulation No: 165/2006 (Amended by $1126 / 2007$ ).

AOAC. 2000 AOAC Official Method 992.06., 985.30. RBiopharm Art. No.: P1002.

AOAC. 2005. AOAC Official methods of analysis, William Horwitz, editor; George W. Latimer, Jr., assistant editor. 2005, 999, 07, ISBN; 0.935.584.757 9.780.935.584.752. 999,985.35, (999.07)
Ayan HY, Artık NTD. 2010. Production of tomatoes (Lycopersitcum esculentum) dried in sun and artificial dryer and determination of the changes during the process. Doctoral dissertation, Ankara University Institute of Science, Department of Food Engineering.

Bonazzi C, Dumoulin, E. 2011. Quality changes in food materials as influenced by drying processes. Modern drying technology, 3, 1-20. doi:10.1002/9783527631728.ch14

Barrett DM, Lloyd B. 2012. Advanced preservation methods and nutrient retention in fruits and vegetables. Journal of Science Food Agriculture, 92(1):7-22. doi:10.1002/jsfa.4718.

Cemeroglu B, Yemenicioğlu A, Ozkan M. 2009. Meyve ve Sebze Bileşimi. Meyve ve Sebze İşleme Teknolojisi, (B. Cemeroğlu, ed.) 1. Cilt, s. 1-236. Gıda Teknolojisi Derneği Yayınları No: 39, Bizim Grup Basımevi, Ankara.

Çağlarirmak N. 2006. Ochratoxin A, Hydroxymethylfurfural and Vitamin C levels of sun-dried grapes and sultanas. Journal of food processing and preservation, 30(5), 549-562. https://doi.org/10.1111/j.1745-4549.2006.00088.x

Çağlarırmak N. 2007. Nutrients of exotic mushrooms (L. edodes and Pleurotus species) and estimated approach to the volatile compounds. Food Chemistry, 105, 1188-1194. https://doi.org/10.1016/j.foodchem.2007.02.021

Çağlarırmak N, Hepçimen AZ. 2013. An investigation of nutritional values of dried vegetables. GIDA/The Journal of food, 38(6), 327-333. doi: 10.5505/gida.2013.57441

Çoksöyler N. 1999. Farklı yöntemlerle kurutulan kırmızı biberlerde Aspergillus flavus gelişimi ve aflatoksin oluşumunun incelenmesi, Gıda, Food, 24, (5), 297-306.

Debra RK, O’Neil Jones JM. 2011. Dried fruit consumption is associated with improved diet quality and reduced obesity in US adults: National Health and Nutrition examination survey, 1999-2004., Nutrition Research, 31, 2011, 460-467. doi: 10.1016/j.nutres.2011.05.009

Demirbaş A. 2010. Oil, micronutrients and heavy metal contents of tomatoes, Food Chemistry, 118, 2010, 504- 507. doi:10.1016/j.foodchem.2009.05.007

Doymaz I. 2007. Air-drying characteristics of tomatoes. Journal of Food engineering, 78(4), 1291-1297. https://doi.org/ 10.1016/j.jfoodeng.2005.12.047

Eim VS, Urrea D, Rossello C, Vicente Garcia-Perez J, Femenia A, Simal S. 2013. Optimization of the Drying Process of Carrot (Daucus carota v. Nantes) on the Basis of Quality Criteria. Dry Technology 31(8):951-962. doi:10.1080/ 07373937.2012.707162

Finglas PM, Foulks RM. 1984. Determination of B Vitamins in Tablets (version) 1.2 Roche Vitamins Ltd. P. Hofmann, C. Brodhag, W. Schüep). Food Chemistry, (15), 37-44.

Giovanelli G, Zanoni B, LAvelli V, NAni R. 2002. Water sorption, drying and antioxidant properites of dried tomato products. Journal of Food Engineering, 52, 2002, 135-141. https://doi.org/10.1016/S0260-8774(01)00095-4

Gould GW. 2012. New methods of food preservation. Springer Science Business Media. ISBN 978-1-4615-2105-1

Gökmen V, Kahraman N, Demir N, Acar J. 2000. Enzymatically validated liquid chromatographic method for the determination of ascorbic and dehydroascorbic acids in fruit and vegetables, Journal Chromatography A, Volume 881, Issues 1-2,9, June 2000, pages 309-316. doi: 10.1016/s00219673(00)00080-7

Hnin KK, Zhang M, Mujumdar AS, Zhu, Y. 2018. Emerging food drying technologies with energy-saving characteristics: A review. Drying Technology.37:12, 1465-1480, doi: 10.1080/07373937.2018.1510417.

Hui YH. (Ed.) 2006. Handbook of food science, technology, and engineering (Vol. III). CRC press. ISBN 9780849398476 https://doi.org/10.1201/b15995

Kaur A, Kaur D, Oberoi DPS, Gill BS, Sogi DS. 2008. Effect of dehydration on physicochemical properties of mustard, mint and spinach. Journal of Food Process Preservation, 32(1): 103-116. https://doi.org/10.1111/j.1745-4549.2007.00168.x 
Kamman JF, Wanthesen JJ, Labuza TP. 1980. Technique for measuring thiamin and riboflavin in fortified foods. Journal of Food Science, 45, 1497-1499.

Li J, Li Z, Wang N, Raghavan GSV, Pei Y, Song C, Zhu G. 2020. Novel sensing technologies during the food drying process. Food Engineering Reviews, 1-28. https://doi.org/ 10.1007/s12393-020-09215-2

Louka N, Juhel F, Allaf K. 2004. Quality studies on various types of partially dried Vegetables texturized by Controlled Sudden Decompression: General patterns for the Variation of the expansion ratio. Journal of Food Engineering.65 (2): 245 253. doi:10.1016/j.jfoodeng.2004.01.021

Jin W, Mujumdar AS, Zhang M, Shi W. 2017. Novel Drying Techniques for Spices and Herbs: A Review. Food Engineering Review. 2017(1), 1-12. doi:10.1007/s12393017-9165-7

Manz U, Philipp K. 1998. Analytical Methods for Vitamins and Caratonoids in Feed. Animal Nutrition and Health Vitamins and Fine Chemicals Division. (42) Roche 1998.

Mayer-Miebach E, Behsnilian D, Regier M, Schuchmann HP 2005. Thermal processing of carrots: lycopene stability and isomerisation with regard to antioxidant potential. Food Res Int. 38(8-9): 1103-1108. DOİ: 10.1016/j.foodres.2005.03.018

Minhthy LN, Steven JS. 1999. Lycopene: chemical and biological properties. Food Technology, 53(2), 38-45.

Miranda M, Maureira H, Rodriguez K, Vega-Gálvez A. 2009. Influence of temperature on the drying kinetics, physicochemical properties, and antioxidant capacity of Aloe Vera (Aloe Barbadensis Miller) gel. Journal of Food Engineering, 91(2), 297-304. doi:10.1016/j.jfoodeng. 2008.09.007

Moses JA, Norton T, Alagusundaram K, Tiwari BK. 2014a. Novel drying techniques for the food industry. Food Engineering Review, 6:43-55. https://doi.org/10.1007/ s12393-014-9078-7

Oliveira SM, Brandao TRS, Silva CLM. 2016. Influence of drying processes and pretreatments on nutritional and bioactive characteristics of dried vegetables: a review. Food Engineering Review, 8:134-163. DOİ10.1007/s12393-0159124-0

Okos MR, Narsimhan G, Singh RK, Witnauer AC. 1992. Food dehydration. In D. R. Heldman D. B. Lund (Eds.), Handbook of food engineering. New York: Marcel Dekker.
Nguyen M, Schwartz SJ. 1999. Lycopene: Chemical and biological properties. Food Technology, 53 (2) pp. 38-45.

Pazir F, Yurdagel Ü, Ural A, Babalik Ö. 1996. Factors affecting sulphur dioxide absorption in tomatoes prepared for sun drying. In 3rd Karlsruhe Nutrition Symposium European Research towards Safer and Better Food (p. 89).

Paolo D, Bianchi G, Morelli CF, Speranza G, Campanelli G, Kidmose U, Scalzo RL. 2019. Impact of drying techniques, seasonal variation and organic growing on flavor compounds profiles in two Italian tomato varieties. Food chemistry, 298, 125062. https://doi.org/10.1016/j.foodchem.2019.125062

Rhone Diagnostics Technologies, 1999. Cereal Ochratoxin A extraction method, Application notes for analysis of Ochratoxin A in cereal using sodium bicarbonate extraction in conjunction with Ochrap. Application note. Ref NA9P14VI.

Singh S, Shalini R. 2016. Effect of Hurdle Technology in Food Preservation: A Review, Critical Reviews in Food Science and Nutrition, 56:4, 641-649, doi:10.1080/10408398.2012. 761594

Şahin FH, Ülger P, Aktaş T, Hülya ORAK. 2010. Effects of Different Drying Techniques on Some Nutritional Components of Tomato (Lycopersicon esculentum). Tarım Makinaları Bilimi Dergisi, 6(1), 71-78. Retrieved from https://dergipark.org.tr/tr/pub/tarmak/issue/11543/137525

Sencer E. 1983. Beslenme ve Diyet. İstanbul Üniversitesi Bayda yayınlar11983No; 4, İstanbul, 102-215.

Şen L, Nas S. 2010. Kuru incir, üzüm ve kırmızı biberlerde mikotoksin varlığ 1 , Akademik G1da (3), 2010, 24-32. Retrieved from https://dergipark.org.tr/tr/pub/akademikgida/issue/55833/764791

Rahman MS, Perera CO. 2004. Drying and food preservation. Food science and technology, New York-Marcel Dekker-, $167,403$.

Zakaria M, Simpson K, Brown PR, Krstulovic A. 1979. Use of reversed-phase high-performance liquid chromatographic analysis for the determination of provitamin A carotenes in tomatoes. Journal of Chromatography A, 176(1), 109-117. doi:10.1016/s0021-9673(00)92091-0

Willis MS, Wians FH. 2003. The role of nutrition in preventing prostate cancer: a review of the proposed mechanism of action of various dietary substances, Clinica Chimica Acta 330. 57-83. doi: 10.1016/s0009-8981(03)00048-2 